EPJ manuscript No.

(will be inserted by the editor)

\title{
Pycnonuclear reactions in dense stellar matter
}

\author{
D.G. Yakovlev ${ }^{1}$, K.P. Levenfish ${ }^{1}$, and O.Y. Gnedin ${ }^{2}$ \\ 1 Ioffe Physical Technical Institute, Politekhnicheskaya 26, St.-Petersburg, 194021, Russia \\ 2 Ohio State University, 760 1/2 Park Street, Columbus, OH 43215, USA \\ Received: date / Revised version: date
}

\begin{abstract}
We discuss pycnonuclear burning of highly exotic atomic nuclei in deep crusts of neutron stars, at densities up to $10^{13} \mathrm{~g} \mathrm{~cm}^{-3}$. As an application, we consider pycnonuclear burning of matter accreted on a neutron star in a soft X-ray transient (SXT, a compact binary containing a neutron star and a low-mass companion). The energy released in this burning, while the matter sinks into the stellar crust under the weight of newly accreted material, is sufficient to warm up the star and initiate neutrino emission in its core. The surface thermal radiation of the star in quiescent states becomes dependent of poorly known equation of state (EOS) of supranuclear matter in the stellar core, which gives a method to explore this EOS. Four qualitatively different model EOSs are tested against observations of SXTs. They imply different levels of the enhancement of neutrino emission in massive neutron stars by (1) the direct Urca process in nucleon/hyperon matter; (2) pion condensates; (3) kaon condensates; (4) Cooper pairing of neutrons in nucleon matter with the forbidden direct Urca process. A low level of the thermal quiescent emission of two SXTs, SAX J1808.4-3658 and Cen X-4, contradicts model (4). Observations of SXTs test the same physics of dense matter as observations of thermal radiation from cooling isolated neutron stars, but the data on SXTs are currently more conclusive.
\end{abstract}

PACS. 97.60.Jd Neutron stars - 26.60.+c Nuclear matter aspects of neutron stars

\section{Introduction}

Neutron stars (NSs) are the most compact stars known in the Universe. Their masses are $M \sim 1.4 M_{\odot}\left(M_{\odot}\right.$ being , the solar mass) while their radii are $R \sim 10 \mathrm{~km}$. They are thought to consist (e.g., Ref. [1 2]) of a massive dense core surrounded by a thin crust (of mass $\lesssim 0.01 M_{\odot}$ and thickness $\lesssim 1 \mathrm{~km}$ ). The crust-core interface occurs at the density $\rho \approx \rho_{0} / 2$, where $\rho_{0}=2.8 \times 10^{14} \mathrm{~g} \mathrm{~cm}^{-3}$ is the standard density of saturated nuclear matter.

The NS crust is composed of neutron-rich atomic nuclei, strongly degenerate electrons and (at $\rho \gtrsim 4 \times 10^{11}$ $\mathrm{g} \mathrm{cm}^{-3}$ ) of free neutrons dripped from nuclei. NS cores contain degenerate matter of supranuclear density (up to $\left.10-15 \rho_{0}\right)$. Its composition and equation of state (EOS) are model dependent (being determined by still poorly known strong interactions in dense matter). On the other hand, these properties are almost not constrained by observations (e.g., Ref. 1]). The NS core is usually subdivided into the outer core $\left(\rho \lesssim 2 \rho_{0}\right)$ and the inner core $\left(\rho \gtrsim 2 \rho_{0}\right)$. The outer core is available in all NSs, while the inner core is present only in massive, more compact NSs. The outer core is thought to be composed of neutrons, with the admixture of protons, electrons (and possibly muons). The composition and EOS of the inner core

Send offprint requests to: is still a mystery. It may be the same composition as in the outer core, with a possible addition of hyperons. Alternatively, the inner core may contain pion- or kaon condensates, or quark matter, or the mixture of these components. Another complication is introduced by superfluidity of neutrons, protons and other baryons in dense matter. Superfluid gaps are very model dependent (e.g., Ref. [3]).

It is unlikely that the fundamental problem of the EOS of supranuclear matter can be solved on purely theoretical grounds. However the solution can be obtained by comparing theoretical models of NSs with observations. We will mention two lines of such studies. The first one is based on theory and observations of cooling isolated NSs (e.g., Refs. [4, 5 6. 6]). These studies, carried out over several decades, have constrained the properties of dense matter but have not solved the problem (Sect. 4). Here, we focus on an alternative method to test the same physics on another class of objects, transiently accreting NSs in soft X-ray transients (SXTs). This method is based on pycnonuclear burning of accreted matter. The method is new but seems to be currently more restrictive.

\section{Deep crustal heating}

Nuclear fusion reactions in normal stars proceed in the thermonuclear regime, in which the Coulomb barrier is 
D.G. Yakovlev et al.: Pycnonuclear reactions in dense stellar matter

penetrated owing to the thermal energy of colliding nuclei. Here, we discuss another, pycnonuclear regime where the Coulomb barrier is penetrated due to zero-point vibrations of nuclei arranged, for instance, in a lattice. The thermonuclear regime is realized in a rather low-density and warm plasma, whereas the pycnonuclear regime operates at high densities and not too high temperatures. Pycnonuclear reactions are almost temperature independent and occur even at $T=0$. They were suggested by Gamow 8] in 1938. The first calculations of pycnonuclear reaction rates were done by Wildhack 9] upon Gamow's request. The strict approach for calculating these rates was formulated by Salpeter and van Horn [10] who studied also three other regimes of nuclear burning in dense matter (the thermonuclear regime with strong enhancement due to plasma screening effects; the intermediate thermopycno nuclear regime; and the thermally enhanced pycnonuclear regime). The pycnonuclear regime has been analyzed later in a number of publications (e.g., 11,12 and references therein). Pycnonuclear reactions are extremely slow at densities $\rho$ typical for normal stars but intensify with increasing $\rho$. For example, carbon burns rapidly into heavier elements at $\rho \gtrsim 10^{10} \mathrm{~g} \mathrm{~cm}^{-3}$.

Pycnonuclear burning can be important in transiently accreting NSs. The heat released due to the infall of matter and thermonuclear reactions in the surface layers is radiated away by photons from the NS surface and cannot warm up the NS interiors. The accreted matter sinks into the NS crust under the weight of newly accreted material. The density gradually increases in a matter element, and the nuclei undergo transformations - beta captures, absorption and emission of neutrons, and pycnonuclear reactions. The nuclei evolve then into highly exotic atomic nuclei which are unstable in laboratory conditions but stable in dense matter. The transformations and associated energy release have been studied by Haensel and Zdunik 13. 14. At $\rho \gtrsim 10^{9} \mathrm{~g} \mathrm{~cm}^{-3}$ the transformations are almost temperature independent but depend on the composition of matter at $\rho \sim 10^{9} \mathrm{~g} \mathrm{~cm}^{-3}$. The main energy release occurs at densities from about $10^{12}$ to $10^{13} \mathrm{~g} \mathrm{~cm}^{-3}$, several hundred meters under the NS surface, in pycnonuclear reactions. For iron matter at $\rho \sim 10^{9} \mathrm{~g} \mathrm{~cm}^{-3}$, these reactions are $13{ }^{34} \mathrm{Ne}+{ }^{34} \mathrm{Ne} \rightarrow{ }^{68} \mathrm{Ca} ;{ }^{36} \mathrm{Ne}+{ }^{36} \mathrm{Ne} \rightarrow{ }^{72} \mathrm{Ca}$; and ${ }^{48} \mathrm{Mg}+{ }^{48} \mathrm{Mg} \rightarrow{ }^{96} \mathrm{Cr}$. The total energy release is then $\approx 1.45$ $\mathrm{MeV}$ per accreted baryon; the total NS heating power is determined by the mass accretion rate $\dot{M}$ :

$$
\begin{aligned}
L_{\mathrm{dh}} & =1.45 \mathrm{MeV} \dot{M} / m_{\mathrm{N}} \\
& \approx 8.74 \times 10^{33} \dot{M} /\left(10^{-10} M_{\odot} \mathrm{yr}^{-1}\right) \mathrm{erg} \mathrm{s}^{-1},
\end{aligned}
$$

where $m_{\mathrm{N}}$ is the nucleon mass. It produces deep crustal heating of the star. This heat is spread by thermal conductivity over the entire NS and warms it up.

\section{Thermal states of soft X-ray transients}

It is possible that the deep crustal heating manifests itself in NSs which enter SXTs, compact binaries with low-mass companions [15. These objects undergo the periods of outburst activity (days-months, sometimes years) superimposed with the periods of quiescence (months-decades). An active period is thought to be associated with an accretion of matter from a companion through an accretion disk. The accretion energy released at the NS surface is so large that a SXT looks like a bright X-ray source with the luminosity $L_{X} \sim 10^{36}-10^{38} \mathrm{erg} \mathrm{s}^{-1}$. The accretion is switched off or suppressed in quiescent periods when $L_{X}$ drops below $10^{34} \mathrm{erg} \mathrm{s}^{-1}$. In many cases, the spectra of quiescent emission contain the thermal component, well described by NS hydrogen atmosphere models with effective surface temperatures from few $10^{5} \mathrm{~K}$ to $\sim 10^{6} \mathrm{~K}$. This can be the radiation emergent from warm NSs.

The suggestion to interpret the quiescent radiation spectra of SXTs with hydrogen atmosphere models was put forward by Brown et al. 16] who assumed also that NSs in SXTs could be warmed up by the deep crustal heating of accreted matter. It is important that the crustal heat is partly radiated away by neutrinos from the entire NS body. Because the neutrino luminosity depends on the NS structure, the remaining heat, diffused to the surface and radiated away by photons, becomes also dependent of the NS structure. This opens an attractive possibility (see [18, 19,20,21 and references therein) to explore the NS structure by comparing the observed quiescent thermal radiation from SXTs with theoretical predictions.

We outline the results of such studies using the theory of thermal states of transiently accreting NSs described in Ref. 22]. These stars are thermally inertial, with thermal relaxation times $\sim 10^{4}$ yr $[19$. In the first approximation, a transiently accreting NS is in a (quasi)stationary steady state determined by the mass accretion rate $\dot{M} \equiv\langle\dot{M}\rangle$ averaged over thermal relaxation time-scales. Typically, $\langle\dot{M}\rangle$ ranges from $10^{-14}$ to $10^{-9} M_{\odot} \mathrm{yr}^{-1}$ and does not increase noticeably the NS mass during long periods of NS evolution. Quasistationary states are expected to be rather insensitive to variations of the accretion rate associated with a sequence of active and quiescent states.

Thermal states of accreting NSs can be greatly affected by the neutrino emission from NS interiors (mainly from the cores). The neutrino luminosity of the star, $L_{\nu}$, in its turn, can strongly depend on the NS mass (as schematically shown in Fig. 11). Low-mass NSs possess nucleon cores with not too high neutrino emission determined mainly by the modified Urca (Murca) and nucleon-nucleon bremsstrahlung (brems) processes. High-mass NSs, in addition to the outer nucleon cores, possess the inner cores whose composition and EOS are unknown (Sect. 1). Their neutrino emission can be enhanced with respect to the emission of low-mass NSs. Four qualitatively different enhancement mechanisms are (e.g., Ref. 7]): (1) a very powerful direct Urca (Durca) process in the cores of massive NSs containing nucleons (and possibly hyperons); (2) a less powerful direct-Urca-like process in pion-condensed cores; (3) even less powerful similar processes in kaon-condensed or quark NS cores; (4) even weaker enhancement of the neutrino emission owing to mild Cooper pairing of neutrons in the nucleon inner NS cores with forbidden direct 


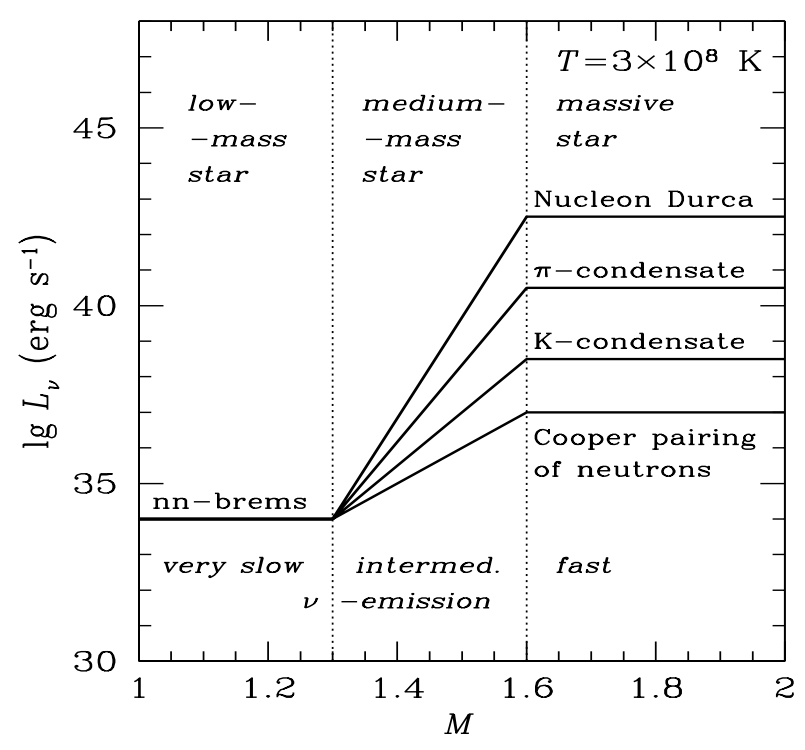

Fig. 1. A sketch of the NS neutrino luminosity $L_{\nu}$ versus NS mass at the internal stellar temperature $T=3 \times 10^{8} \mathrm{~K}$ for four scenarios of NS structure (from Ref. [7).

Urca process [5] 6]. A transition from the slow neutrino emission in low-mass NSs to a fast emission in massive NSs with increasing $M$ occurs in medium-mass NSs; the mass range of medium-mass stars is model-dependent. The NS neutrino luminosity is a strong function of the internal stellar temperature.

A thermal state of the star is determined by the thermal balance equation (which implies that the crustal heating power is equal to the sum of photon and neutrino luminosities). Solving this equation, one obtains a heating curve, the dependence of the thermal photon luminosity (as detected by a distant observer) on the mean mass accretion rate, $L_{\gamma}^{\infty}(\dot{M})$.

Several examples are presented in Fig. 2. The upper curve is calculated for a low-mass NS $\left(M=1.1 M_{\odot}\right)$ whose core is composed of neutrons, protons and electrons. In the core we use a moderately stiff EOS proposed in Ref. 17 (the same version as employed in Ref. 4]). It is assumed that the core contains strongly superfluid protons. Superfluidity suppresses the modified Urca process, the star has a low neutrino luminosity due to neutronneutron bremsstrahlung and stays relatively warm. The lowest curve in Fig. 2 corresponds to the maximum-mass NS $\left(M=1.977 M_{\odot}\right)$ with the same EOS. This star has a massive inner core where the direct Urca process operates and proton superfluidity dies out. The neutrino luminosity becomes exceptionally high, and the star is very cold. The next two curves above the lowest one are schematic models 22] of high-mass NSs with pion-condensed or kaoncondensed cores. The next curve is calculated for a highmass $\left(M=2.05 M_{\odot}\right)$ star, where the direct Urca process is forbidden but the neutrino emission is enhanced by mild Cooper pairing of neutrons in the inner core (the same EOS [23 and model for superfluidity as in Fig. 1 of Ref. [6]).

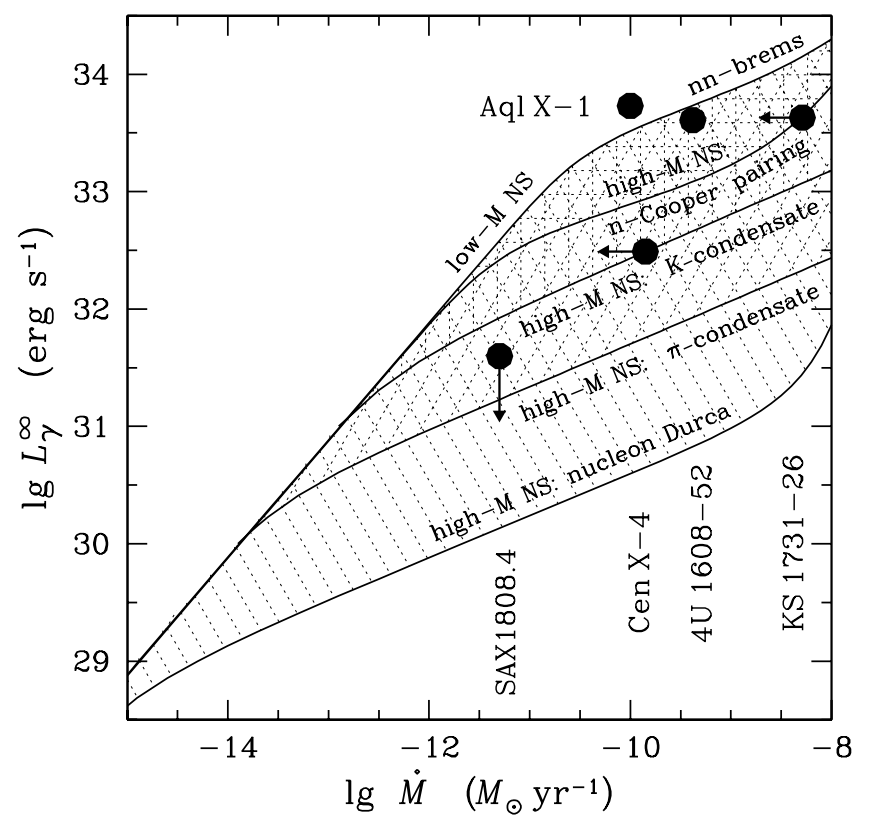

Fig. 2. Quiescent thermal luminosity of several NSs in SXTs versus mass accretion rate compared with theoretical heating curves. Four ranges of $L_{\gamma}^{\infty}$ (different hatching types) correspond to four scenarios of neutrino emission (Fig. 1).

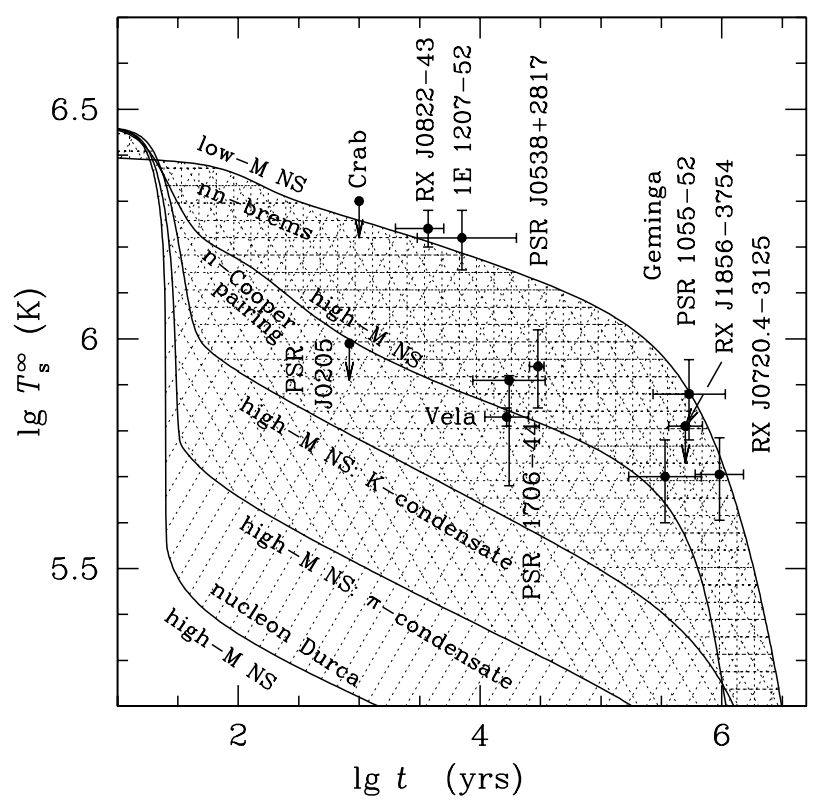

Fig. 3. Effective surface temperatures $T_{\mathrm{s}}^{\infty}$ of several isolated NSs versus their ages compared with theoretical cooling curves (from Ref. [7). Four ranges of $T_{\mathrm{s}}^{\infty}$ (different hatching types) correspond to four scenarios of neutrino emission (Fig. 1).

The heating curve of a low-mass NS provides an upper limit of $L_{\gamma}^{\infty}$, whereas a heating curve of a high-mass NS gives a lower limit of $L_{\gamma}^{\infty}$, for a fixed scenario of neutrino emission. Varying the NS mass from the lower values to the higher we obtain a family of heating curves which fill in the (hatched) space between the upper and lower curves. In Fig. 2 we have four hatched (acceptable) regions for four scenarios of neutrino emission in Fig. 1. 
D.G. Yakovlev et al.: Pycnonuclear reactions in dense stellar matter

\section{Discussion}

These results can be compared with observations of SXTs. The observational data are mostly the same as in Ref. [22]. We regard $L_{\gamma}^{\infty}$ as the thermal quiescent luminosity of NSs, and $\dot{M}$ as the time-averaged mass accretion rate. The value of $\dot{M}$ for KS $1731-26$ is most probably an upper limit. No quiescent thermal emission has been detected 24] from SAX J1808.4-3658, and we present the upper limit of $L_{\gamma}^{\infty}$ for this source. The limit was obtained by P. Stykovsky (private communication, 2004) and discussed in the note added in proof of Ref. 4. The NS seems to be cold, but the result should be taken with caution because it is based on one observation (March 24, 2001) with poor statistics. All the data are rather uncertain and are thus plotted by thick dots.

According to Fig. 2, we can treat NSs in 4U 1608-52 and Aql X-1 as low-mass NSs (the observations of Aql X-1 can be explained [25] assuming the presence of light elements on the NS surface, that increases $\left.L_{\gamma}^{\infty}\right)$. NSs in Cen X-4 and SAX J1808.4-3658 seem to require the enhanced neutrino emission and are thus more massive. The status of the NS in KS 1731-26 is less certain [22] because of poorly determined $\dot{M}$. Similar conclusions have been made with respect to some of these sources or selected groups (see, e.g., [18-25] and references therein) although four scenarios (1)-(4) are discussed together for the first time.

As seen from Fig. 2, the data are still not restrictive. They cannot allow us to discriminate between scenarios (1)-(3) (nucleon NS cores with the direct Urca process, pion-condensed or kaon condensed cores). Nevertheless, the observations of Cen X-4 and especially of SAX J1808.4-3658 seem to contradict scenario (4) of nucleon matter with the forbidden direct Urca process [and the data on SAX J1808.4-3658 marginally contradict scenario (3)].

The same four scenarios can also be tested by comparing observations of thermal radiation of cooling (isolated) NSs with theoretical cooling curves (the dependence of the effective surface temperatures $T_{\mathrm{s}}^{\infty}$, redshifted for a distant observer, on the stellar age). These results are presented in Fig. 3; the data and curves are taken from Ref. 7]. By comparing Figs. 2 and 3 we see that the data on isolated NSs, although more numerous, are currently less conclusive and do not discriminate between all scenarios (1)-(4) [although a discovery of a not too old isolated NS slightly colder than the Vela pulsar would contradict scenario (4)].

Thus, the pycnonuclear burning of highly exotic atomic nuclei in accreting NSs is helpful to solve the longstanding problem of the EOS of supranuclear matter in NS cores. Hopefully, new observations of SXTs and cooling isolated NSs will appear in the near future. Combined together, they will give more stringent constraints on the EOS of dense matter.

The authors are grateful to P. Haensel, M.E. Gusakov, A.D. Kaminker, A.Y. Potekhin, and Yu.A. Shibanov for discussions, and to M.G. Revnivtsev and P. Stykovsky for reprocessing the observations of SAX J1808.4-3658. DGY is grateful to JINA and the organizers of ENAM-2004 for financial support which allowed him to attend the conference. KL acknowledges the support of the Russian Science Support Foundation. This work was supported in part by the RBRF (grants Nos. 02-02-17668 and 03-07-90200) and the RLSS (grant 1115.2003.2).

\section{References}

1. J.M. Lattimer and M. Prakash, Astrophys. J. 550 (2001) 426.

2. P. Haensel, in: Final Stages of Stellar Evolution, C. Motch and J.-M. Hameury (eds.), EAS Publ. Ser.: EDP Sci. (2003) 249 .

3. U. Lombardo and H.-J. Schulze, in: Physics of Neutron Star Interiors, D. Blaschke, N.K. Glendenning, and A. Sedrakian (eds.), Springer: Berlin (2001) 30.

4. D.G. Yakovlev and C.J. Pethick, Ann. Rev. Astron. Astrophys. 42 (2004) 169.

5. D. Page, J.M. Lattimer, M. Prakash, and A.W. Steiner, Astroph. J. Suppl. 155 (2004) 623.

6. M.E. Gusakov, A.D. Kaminker, D.G. Yakovlev, and O.Y. Gnedin, A\&A 423 (2004) 1063.

7. D.G. Yakovlev, O.Y. Gnedin, M.E. Gusakov, A.D. Kaminker, K.P. Levenfish, and A.Y. Potekhin, in: Proc. Intern. Nucl. Phys. Conf. (Göteborg, Sweden, June 27 July 2, 2004), in press astro-ph/0409751.

8. G. Gamow, Phys. Rev. 55 (1939) 718.

9. W.A. Wildhack, Phys. Rev. 57 (1940) 81.

10. E.E. Salpeter and H.M. van Horn, Astrophys. J. 155 (1969) 183.

11. S. Schramm and S.E. Koonin, Astrophys. J. 365 (1990) 296; erratum: Astrophys. J. 377 (1991) 343.

12. H. Kitamura, Astrophys. J. 539 (2000) 888.

13. P. Haensel and J.L. Zdunik, A $\& A 227$ (1990) 431.

14. P. Haensel and J.L. Zdunik, A\&A 404 (2003) L33.

15. W. Chen, C.R. Shrader and M. Livio, Astrophys. J. 491 (1997) 312.

16. E.F. Brown, L. Bildsten and R.E. Rutledge, Astrophys. J. Lett. 504 (1998) L95.

17. M. Prakash, T.L. Ainsworth and J.M. Lattimer, Phys. Rev. Lett. 61 (1988) 2518.

18. G. Ushomirsky and R.E. Rutledge, MNRAS 325 (2001) 1157.

19. M. Colpi, U. Geppert, D. Page and A. Possenti, Astrophys. J. Lett. 548 (2001) L175.

20. R.E. Rutledge, L. Bildsten, E.F. Brown, G.G. Pavlov, V.E. Zavlin and G. Ushomirsky, Astrophys. J. 580 (2002) 413.

21. E.F. Brown, L. Bildsten and P. Chang, Astrophys. J. 574 (2002) 920.

22. D.G. Yakovlev, K.P. Levenfish and P. Haensel, A\&A 407 (2003) 265.

23. F. Douchin and P. Haensel, A\&A 380 (2001) 151.

24. S. Campana, L. Stella, F. Gastaldello, S. Mereghetti, M. Colpi, G.L. Israel, L. Burderi, T. Di Salvo and R.N. Robba, Astrophys. J. Lett. 575 (2002) L15.

25. D.G. Yakovlev, K.P. Levenfish, A.Y. Potekhin, O.Y. Gnedin, and G. Chabrier, A\& A 417 (2004) 169. 